\title{
Far field of beams generated by quasi-homogeneous sources passing through polarization gratings
}

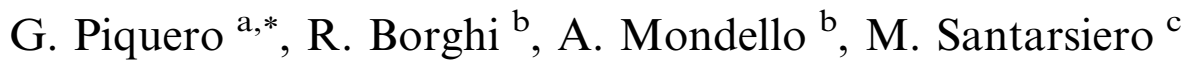 \\ a Departamento de Óptica, Facultad de Ciencias Fisicas, Universidad Complutense de Madrid, Ciudad Universitaria s/n, \\ E-28040 Madrid, Spain \\ b Dipartimento di Ingegneria Elettronica, Università "Roma Tre” and Istituto Nazionale per la Fisica della Materia, \\ Via della Vasca Navale 84, I-00146 Rome, Italy \\ "Dipartimento di Fisica, Università "Roma Tre” and Istituto Nazionale per la Fisica della Materia, Via della Vasca Navale 84, \\ I-00146 Rome, Italy
}

Received 26 February 2001; accepted 5 June 2001

\begin{abstract}
We analyze the polarization features of the beam, generated by a class of partially polarized quasi-homogeneous sources, which propagates through a polarization grating. Analytical expressions in the far zone for the beam coherence polarization matrix, the degree of polarization and the Stokes parameters are given. In particular, it is shown that, under some hypotheses, it is possible to completely and uniformly depolarize the beam in the far field. The influence of source parameters, such as the state of polarization, intensity and degree of coherence, on the degree of polarization and the Stokes parameters is also investigated. @ 2001 Elsevier Science B.V. All rights reserved.
\end{abstract}

Keywords: Polarization; Gratings; Coherence; Sources

\section{Introduction}

It is well known that degree and state of polarization of uniformly fully polarized beams remain constant upon free propagation. This behavior is not followed, in general, by beams with partial polarization and/or with a non-uniform state of polarization across their transversal sections. This kind of beams have currently gained an increasing interest [1-5] not only from the theoretical point of view but also experimentally. In this sense, some

\footnotetext{
${ }^{*}$ Corresponding author. Fax: +34-1-394-4683.

E-mail address: piquero@eucmax.sim.ucm.es (G. Piquero).
}

authors investigated the polarization features after propagation of beams generated by incoherent sources [6-8] or by Gaussian Schell-model (GSM) sources [9-11].

On the other hand, some kind of anisotropic elements, such as polarizing filters whose transmission axis changes periodically along a line (called polarization gratings, PG), have been considered for several applications [12-15]. One of the applications concerns the possibility of synthesizing beams endowed with special polarization characteristics [8].

Up to now, PGs have been studied for the case of fully coherent incident beams. In the present paper, the formalism of the beam coherence 


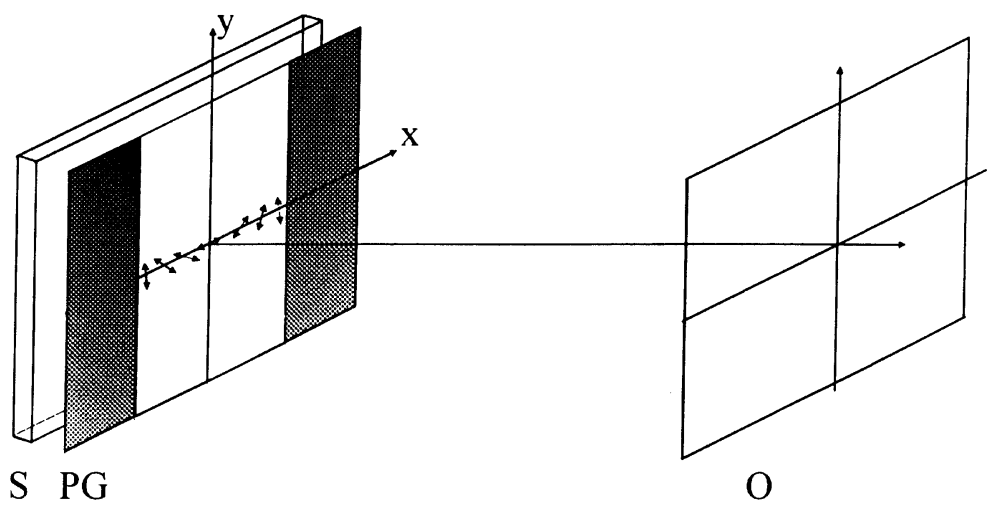

Fig. 1. Geometry of the problem (S: initial source, PG: polarization grating, O: observation plane).

polarization $(\mathrm{BCP})$ matrix $[16,17]$ is used to investigate beams radiated by partially polarized quasi-homogeneous (Q-H) sources [18] and propagating after a PG. We recall that for a Q-H source the intensity distribution of light varies slowly with position, remaining sensibly constant over distances of the order of the correlation length of the light vibration across the source plane. They have been extensively used in the scalar framework, without taking the polarization of light into account, and important results have already been obtained. For instance, it has been proved that they can generate highly directional beams, although they may be rather incoherent from the spatial viewpoint $[19,20]$.

In this work we first consider two classes of partially coherent partially polarized Q-H planar sources, namely, those obtained as incoherent superpositions of two orthogonally totally polarized sources. For the first class, the two constituent sources are linearly polarized along orthogonal directions, say $x$ and $y$. For simplicity, we let such two sources to have the same degree of coherence and the same intensity profile, up to a proportionality factor, so that they are represented by two correlation functions that are proportional to each other. On varying the relative powers of the two sources one can set the degree of polarization of the overall source, ranging from the case of a completely unpolarized source (when the weights are equal) to that of fully linearly polarized sources (when one of the two weights is set to zero). The second class is obtained, in an analogous way, by superimposing mutually uncorrelated sources circularly polarized with opposite helicity. In this way, on acting on the relative weights of the two correlation functions, we can consider all the cases between a completely unpolarized source and fully circularly polarized ones.

The beam radiated by the source obtained in such way (which will be referred to as "initial source") is eventually let varying through a PG. For simplicity, the plane of the PG is chosen as coincident with the plane of the initial source (see Fig. 1) so that propagation from the source plane to the PG needs not to be considered. The light distribution after the PG will constitute a partially coherent partially polarized source, whose propagation features will be the subject of the present paper. In particular, analytical expressions for the resulting BCP matrix, degree and state of polarization in the far zone are given as functions of the period of the grating and of the intensity, degree of coherence, and polarization state of the initial source. It will be shown that, under some hypotheses, the degree of polarization in the far zone is independent of the degree of coherence and is uniform across the transversal section of the beam, taking any value from zero to one. In particular, it is even possible to completely depolarize the beam. This fact suggests a technique to synthesize completely unpolarized beams, which are required in some optical applications [21,22].

As a particular example, a GSM source [18], for which both the intensity and the degree of coherence are Gaussian, is chosen as the initial source. 
In particular, for totally linearly and uniformly polarized GSM sources the minimum value of the polarization degree that can be reached in the far field, is calculated.

The paper is structured as follows: In Section 2 we introduce the formalism to be used. Analytical expressions at the far zone for the BCP, the degree of polarization and the Stokes parameters are obtained in Section 3 for some classes of partially polarized Q-H sources: those which are written as incoherent superpositions of orthogonally linearly polarized or circularly polarized beams propagating through PGs. In order to obtain simple expressions of the previous results, in this section also we consider some hypotheses on the intensity and on the degree of coherence into account. For such example we also investigate the influence of the state of polarization of the initial source on the polarization features at the far zone. In particular we consider the case of linearly totally polarized Q-H sources. In Section 4 the case of GSM sources, that can be considered of the Q-H type only in a particular approximation, is investigated. By means of this example we analyze the meaning of the hypotheses done before. Finally in Section 5 the main results are summarized.

\section{Preliminaries}

In the following we will consider quasi-monochromatic radiation, so that use will be made of the formalism introduced in Refs. [16,17]. Nonetheless, it should be stressed that the same approach could be used for a wide-band source, provided that a space-frequency approach $[10,18]$ is considered. We consider a typical cross section of the beam and denote by $\mathbf{r}_{1}$ and $\mathbf{r}_{2}$ the position vectors of two points. Within the paraxial approximation and assuming that the longitudinal component of the field can be neglected, we work with the BCP matrix, defined as [16,17]

$\hat{J}\left(\mathbf{r}_{1}, \mathbf{r}_{2}\right)=\left(\begin{array}{ll}J_{x x}\left(\mathbf{r}_{1}, \mathbf{r}_{2}\right) & J_{x y}\left(\mathbf{r}_{1}, \mathbf{r}_{2}\right) \\ J_{y x}\left(\mathbf{r}_{1}, \mathbf{r}_{2}\right) & J_{y y}\left(\mathbf{r}_{1}, \mathbf{r}_{2}\right)\end{array}\right)$,

where

$J_{\alpha \beta}\left(\mathbf{r}_{1}, \mathbf{r}_{2}\right)=\left\langle E_{\alpha}^{*}\left(\mathbf{r}_{1} ; t\right) E_{\beta}\left(\mathbf{r}_{2} ; t\right)\right\rangle$.
Here, each of the suffixes $\alpha$ and $\beta$ can be either $x$ or $y, E_{x}$ and $E_{y}$ are the components of the complex electric field vector in the $x$ and $y$ directions, respectively. Furthermore, the asterisk stands for the complex conjugate and angle brackets denote time average. For such a source the intensity distribution is

$I(\mathbf{r})=\operatorname{Tr}[\hat{J}(\mathbf{r}, \mathbf{r})]$,

the degree of polarization in $\mathbf{r}$ is calculated as [23]

$\mathscr{P}(\mathbf{r})=\sqrt{1-\frac{4 \operatorname{det}[\hat{J}(\mathbf{r}, \mathbf{r})]}{\{\operatorname{Tr}[\hat{J}(\mathbf{r}, \mathbf{r})]\}^{2}}}$,

and the Stokes parameters [23] turn out to be

$s_{0}(\mathbf{r})=J_{x x}(\mathbf{r}, \mathbf{r})+J_{y y}(\mathbf{r}, \mathbf{r})$,

$s_{1}(\mathbf{r})=J_{x x}(\mathbf{r}, \mathbf{r})-J_{y y}(\mathbf{r}, \mathbf{r})$,

$s_{2}(\mathbf{r})=2 \operatorname{Re}\left[J_{x y}(\mathbf{r}, \mathbf{r})\right]$,

$s_{3}(\mathbf{r})=2 \operatorname{Im}\left[J_{x y}(\mathbf{r}, \mathbf{r})\right]$,

where $\operatorname{Tr}$ and det stand for the trace and determinant of a matrix.

It may be useful to recall the general expressions of the BCP matrices representing light fields with particular polarization states. For the case of linear polarization along the $x$ - and the $y$-axis, for instance, we have

$\hat{J}_{X}\left(\mathbf{r}_{1}, \mathbf{r}_{2}\right)=J_{X}\left(\mathbf{r}_{1}, \mathbf{r}_{2}\right)\left(\begin{array}{ll}1 & 0 \\ 0 & 0\end{array}\right) ;$
$\hat{J}_{Y}\left(\mathbf{r}_{1}, \mathbf{r}_{2}\right)=J_{Y}\left(\mathbf{r}_{1}, \mathbf{r}_{2}\right)\left(\begin{array}{ll}0 & 0 \\ 0 & 1\end{array}\right)$,

respectively, where $J_{X}$ and $J_{Y}$ are standard (scalar) correlation functions pertinent to the two uncorrelated sources. For the case of circularly polarized light fields we have, with analogous meaning of the introduced symbols,

$\hat{J}_{\mathrm{L}}\left(\mathbf{r}_{1}, \mathbf{r}_{2}\right)=\frac{1}{2} J_{\mathrm{L}}\left(\mathbf{r}_{1}, \mathbf{r}_{2}\right)\left(\begin{array}{cc}1 & -\mathrm{i} \\ \mathrm{i} & 1\end{array}\right) ;$
$\hat{J}_{\mathrm{R}}\left(\mathbf{r}_{1}, \mathbf{r}_{2}\right)=\frac{1}{2} J_{\mathrm{R}}\left(\mathbf{r}_{1}, \mathbf{r}_{2}\right)\left(\begin{array}{cc}1 & \mathrm{i} \\ -\mathrm{i} & 1\end{array}\right)$,

for left-handed and right-handed states, respectively. 


\section{Partially polarized Q-H sources}

In the following, for the sake of simplicity, we consider the one-dimensional case. The latter is thought of as the superposition of two mutually uncorrelated orthogonally polarized sources. The cases of linearly polarized and circularly polarized component sources will be treated separately in the following subsections.

\subsection{Case I: Superposition of linearly polarized} sources

In this case the BCP matrix of the resulting source can be written in the form (see Eq. (9))

$\hat{J}_{\mathrm{I}}\left(x_{1}, x_{2}\right)=J_{X}\left(x_{1}, x_{2}\right)\left(\begin{array}{ll}1 & 0 \\ 0 & 0\end{array}\right)+J_{Y}\left(x_{1}, x_{2}\right)\left(\begin{array}{ll}0 & 0 \\ 0 & 1\end{array}\right)$.

As stated above, for simplicity we choose the two functions to be proportional to each other, so that

$J_{X}\left(x_{1}, x_{2}\right)=A J_{\mathrm{s}}\left(x_{1}, x_{2}\right) ; \quad J_{Y}\left(x_{1}, x_{2}\right)=B J_{\mathrm{s}}\left(x_{1}, x_{2}\right)$,

where $A$ and $B$ are positive quantities and $J_{\mathrm{s}}\left(x_{1}, x_{2}\right)$ is a well-defined coherence function. The resulting BCP matrix turns out to be

$\hat{J}_{\mathrm{I}}\left(x_{1}, x_{2}\right)=J_{\mathrm{s}}\left(x_{1}, x_{2}\right)\left(\begin{array}{cc}A & 0 \\ 0 & B\end{array}\right)$.

It is convenient to impose a constrain on the coefficients $A$ and $B$, that is

$A+B=1$,

so that the intensity of the resulting source remains unchanged on varying $A$. On the contrary, it is easily seen that the choice of $A(\in[0,1])$ rules the degree of polarization of the source. The latter, denoted by $\mathscr{P}_{\mathrm{s}}$, in fact, can be evaluated from the general relation in Eq. (4) applied to Eq. (13), yielding

$\mathscr{P}_{\mathrm{s}}=\frac{|A-B|}{A+B}=|2 A-1|, \quad A \in[0,1]$,

where Eq. (14) has been used.
Let now the source be covered by a polarization grating whose polarization angle varies linearly with $x$. Such an optical element, which is sketched in Fig. 1, is represented by a Jones matrix [18] of the type [12]

$\widehat{T}(x)=\left(\begin{array}{cc}C^{2} & C S \\ C S & S^{2}\end{array}\right)$,

where $C=\cos (\pi x / L)$ and $S=\sin (\pi x / L), L$ being the period of the polarizing structure.

The BCP matrix of the emerging field can be evaluated as [17]

$$
\begin{aligned}
\hat{J}\left(x_{1}, x_{2}\right)= & \widehat{T}^{\dagger}\left(x_{1}\right) \hat{J}_{\mathrm{I}}\left(x_{1}, x_{2}\right) \widehat{T}\left(x_{2}\right) \\
= & \frac{1}{2} J_{\mathrm{s}}\left(x_{1}, x_{2}\right)\left(\begin{array}{cc}
C_{1}^{2} & C_{1} S_{1} \\
C_{1} S_{1} & S_{1}^{2}
\end{array}\right)\left(\begin{array}{ll}
A & 0 \\
0 & B
\end{array}\right) \\
& \times\left(\begin{array}{cc}
C_{2}^{2} & C_{2} S_{2} \\
C_{2} S_{2} & S_{2}^{2}
\end{array}\right),
\end{aligned}
$$

where the dagger denotes the Hermitian conjugate and

$C_{j}=\cos \left(\frac{\pi}{L} x_{j}\right) ; \quad S_{j}=\sin \left(\frac{\pi}{L} x_{j}\right) ; \quad(j=1,2)$.

From Eqs. (17) and (18) we find

$$
\begin{aligned}
\hat{J}\left(x_{1}, x_{2}\right)= & J_{\mathrm{s}}\left(x_{1}, x_{2}\right)\left(A C_{1} C_{2}+B S_{1} S_{2}\right) \\
& \times\left(\begin{array}{ll}
C_{1} C_{2} & C_{1} S_{2} \\
C_{2} S_{1} & S_{1} S_{2}
\end{array}\right) .
\end{aligned}
$$

Eq. (19) represents the BCP matrix of the optical field at the output of the PG. Actually, it is the starting point for the successive calculations.

From the latter equation and Eq. (4) it is easily seen that the degree of polarization is unitary at any point at the output of the PG. Of course, this is not surprising, since the effect of the PG is to fix a linear polarization (although with different vibration directions) at any point across its surface. On the other hand, the intensity profile is (see Eq. (3))

$I(x)=I_{\mathrm{s}}(x)\left[A C^{2}(x)+B S^{2}(x)\right]$,

which is proportional to the incident one only if $A=B$, i.e., in the case of a completely unpolarized initial source. 
Now, in order to study how both the degree and the state of polarization change upon free propagation we calculate the BCP of the beam in the far zone, denoted by $\hat{J}^{\infty}$. The propagation features of the beams radiated by the synthesized source (19) can be analytically calculated once the mathematical form of $J_{\mathrm{s}}\left(x_{1}, x_{2}\right)$ is defined. However, as we shall see in a moment, some interesting results can be obtained even from the general form in Eq. (19), on limiting ourselves to the far zone. The matrix elements, say $J_{\alpha, \beta}^{\infty}$ at the far field assume the form [18]

$$
\begin{aligned}
J_{\alpha \beta}^{\infty}\left(p_{1}, p_{2}\right)= & K \int_{-\infty}^{\infty} \int_{-\infty}^{\infty} J_{\alpha \beta}\left(x_{1}, x_{2}\right) \\
& \times \exp \left[-2 \pi \mathrm{i}\left(p_{2} x_{2}-p_{1} x_{1}\right)\right] \mathrm{d} x_{1} \mathrm{~d} x_{2},
\end{aligned}
$$

where $p_{1}$ and $p_{2}$ are spatial frequencies and

$$
K=\frac{\exp \left[\mathrm{i} \pi \lambda z\left(p_{2}^{2}-p_{1}^{2}\right)\right]}{\lambda z}
$$

$\lambda$ being the mean wavelength of the radiation and $z=$ const a typical plane in the far zone. We can write Eq. (21) in a simpler form as

$$
\begin{aligned}
J_{\alpha \beta}^{\infty}\left(P+\frac{Q}{2}, P-\frac{Q}{2}\right)= & K \int_{-\infty}^{\infty} \int_{-\infty}^{\infty} J_{\alpha \beta}\left(s+\frac{t}{2}, s-\frac{t}{2}\right) \\
& \times \exp [2 \pi \mathrm{i}(s P+t Q)] \mathrm{d} s \mathrm{~d} t
\end{aligned}
$$

if the following change of variables is made:

$\left\{\begin{array}{l}s=\frac{x_{1}+x_{2}}{2}, \\ t=x_{1}-x_{2},\end{array} \quad\left\{\begin{array}{l}P=\frac{p_{1}+p_{2}}{2}, \\ Q=p_{1}-p_{2} .\end{array}\right.\right.$

Eq. (23) can be easily evaluated if a correlation function of the Q-H type is chosen for $J_{\mathrm{s}}\left(x_{1}, x_{2}\right)$. In this case we have [18], indeed,

$J_{\mathrm{s}}\left(x_{1}, x_{2}\right)=I_{\mathrm{s}}\left(\frac{x_{1}+x_{2}}{2}\right) \mu\left(x_{1}-x_{2}\right)$,

where $I_{\mathrm{s}}$ is the intensity distribution and $\mu$ is the degree of coherence of the source. Using the variables defined in Eq. (24), together with Eqs.
(19), (23), and (25), the BCP elements at the far field turn out to be

$$
\begin{aligned}
& J_{x x}^{\infty}(P, Q)= \\
& \frac{K}{16}\left\{(2 A-1) \tilde{\mu}(P)\left[\tilde{I}_{\mathrm{s}}\left(Q+\frac{2}{L}\right)+\tilde{I}_{\mathrm{s}}\left(Q-\frac{2}{L}\right)\right]\right. \\
& +4 A \tilde{\mu}(P) \tilde{I}_{\mathrm{s}}(Q)+2 A\left[\tilde{\mu}\left(P-\frac{1}{2 L}\right)+\tilde{\mu}\left(P+\frac{1}{2 L}\right)\right] \\
& \times\left[\tilde{I}_{\mathrm{s}}\left(Q-\frac{1}{L}\right)+\tilde{I}_{\mathrm{s}}\left(Q+\frac{1}{L}\right)\right] \\
& \left.+\tilde{I}_{\mathrm{s}}(Q)\left[\tilde{\mu}\left(P-\frac{1}{L}\right)+\tilde{\mu}\left(P+\frac{1}{L}\right)\right]\right\}, \\
& J_{x y}^{\infty}(P, Q)= \\
& \frac{K}{16 \mathrm{i}}\left\{(2 A-1) \tilde{\mu}(P)\left[\tilde{I}_{\mathrm{s}}\left(Q+\frac{2}{L}\right)-\tilde{I}_{\mathrm{s}}\left(Q-\frac{2}{L}\right)\right]\right. \\
& +2 A\left[\tilde{\mu}\left(P-\frac{1}{2 L}\right)-\tilde{\mu}\left(P+\frac{1}{2 L}\right)\right]\left[\tilde{I}_{\mathrm{s}}\left(Q+\frac{1}{L}\right)\right. \\
& \left.+\tilde{I}_{\mathrm{s}}\left(Q-\frac{1}{L}\right)\right]+\tilde{I}_{\mathrm{s}}(Q)\left[\tilde{\mu}\left(P-\frac{1}{L}\right)-\tilde{\mu}\left(P+\frac{1}{L}\right)\right] \\
& +2\left[\tilde{I}_{\mathrm{s}}\left(Q+\frac{1}{L}\right) \tilde{\mu}\left(P+\frac{1}{2 L}\right)\right. \\
& \left.\left.-\tilde{I}_{\mathrm{s}}\left(Q-\frac{1}{L}\right) \tilde{\mu}\left(P-\frac{1}{2 L}\right)\right]\right\} \text {, } \\
& J_{y x}^{\infty}(P, Q)= \\
& \frac{K}{16 \mathrm{i}}\left\{(2 A-1) \tilde{\mu}(P)\left[\tilde{I}_{\mathrm{s}}\left(Q+\frac{2}{L}\right)-\tilde{I}_{\mathrm{s}}\left(Q-\frac{2}{L}\right)\right]\right. \\
& +2 A\left[\tilde{I}_{\mathrm{s}}\left(Q+\frac{1}{L}\right)+\tilde{I}_{\mathrm{s}}\left(Q-\frac{1}{L}\right)\right] \\
& \times\left[\tilde{\mu}\left(P+\frac{1}{2 L}\right)-\tilde{\mu}\left(P-\frac{1}{2 L}\right)\right] \\
& -\tilde{I}_{\mathrm{s}}(Q)\left[\tilde{\mu}\left(P-\frac{1}{L}\right)-\tilde{\mu}\left(P+\frac{1}{L}\right)\right] \\
& +2\left[\tilde{I}_{\mathrm{S}}\left(Q+\frac{1}{L}\right) \tilde{\mu}\left(P-\frac{1}{2 L}\right)\right. \\
& \left.\left.-\tilde{I}_{\mathrm{s}}\left(Q-\frac{1}{L}\right) \tilde{\mu}\left(P+\frac{1}{2 L}\right)\right]\right\},
\end{aligned}
$$




$$
\begin{aligned}
& J_{y y}^{\infty}(P, Q)= \\
& \quad \frac{K}{16}\left\{(1-2 A) \tilde{\mu}(P)\left[\tilde{I}_{\mathrm{s}}\left(Q+\frac{2}{L}\right)+\tilde{I}_{\mathrm{s}}\left(Q-\frac{2}{L}\right)\right]\right. \\
& \quad+4(1-A) \tilde{\mu}(P) \tilde{I}_{\mathrm{s}}(Q)+2(A-1) \\
& \quad \times\left[\tilde{\mu}\left(P-\frac{1}{2 L}\right)+\tilde{\mu}\left(P+\frac{1}{2 L}\right)\right] \\
& \quad \times\left[\tilde{I}_{\mathrm{s}}\left(Q-\frac{1}{L}\right)+\tilde{I}_{\mathrm{s}}\left(Q+\frac{1}{L}\right)\right] \\
& \left.\quad+\tilde{I}_{\mathrm{s}}(Q)\left[\tilde{\mu}\left(P+\frac{1}{L}\right)+\tilde{\mu}\left(P-\frac{1}{L}\right)\right]\right\},
\end{aligned}
$$

where the tilde denotes the Fourier transformation.

The local degree of polarization at the coordinate $p$ in the far field can be evaluated from Eq. (4) with $\mathbf{r}$ substituted by $p$, and using Eqs. (26)-(29) with $P=p$ and $Q=0$. It follows that it has in general different values at different points, depending on both the intensity and the degree of coherence of the source.

A simpler expression of the degree of polarization, however, can be gained if the following hypotheses are assumed:

(i) $\tilde{\mu}$ is almost constant in the interval between $(P-(1 / L))$ and $(P+(1 / L))$; roughly speaking, this means that the coherence length across the source is much smaller than the grating period. (ii) $I_{\mathrm{s}}$, and consequently $\tilde{I}_{\mathrm{s}}$, are even functions.

Under such hypotheses and on using Eqs. (26)(29) we obtain, for the local BCP matrix at the far zone,

$$
\begin{array}{rl}
J_{x x}^{\infty}(p, 0) \simeq & \frac{\tilde{\mu}(p)}{8}\left[(2 A-1) \tilde{I}\left(\frac{2}{L}\right)+(2 A+1) \tilde{I}(0)\right. \\
& \left.+4 A \tilde{I}\left(\frac{1}{L}\right)\right], \\
J_{y y}^{\infty}(p, 0) \simeq \frac{\tilde{\mu}(p)}{8}\left[(1-2 A) \tilde{I}\left(\frac{2}{L}\right)+(3-2 A) \tilde{I}(0)\right. & \\
& \left.+4(A-1) \tilde{I}\left(\frac{1}{L}\right)\right], \\
J_{x y}^{\infty}(p, 0) \simeq 0 & 0 \\
J_{y x}^{\infty}(p, 0) \simeq 0 .
\end{array}
$$

On inserting Eqs. (30)-(33) into Eq. (4), the polarization degree, say $P_{\mathrm{I}}^{\infty}$, turns out to be

$$
\mathscr{P}_{\mathrm{I}}^{\infty}(A)=\left|\frac{(2 A-1)\left[\tilde{I}(0)+\tilde{I}\left(\frac{2}{L}\right)\right]+2 \tilde{I}\left(\frac{1}{L}\right)}{2\left[\tilde{I}(0)+(2 A-1) \tilde{I}\left(\frac{1}{L}\right)\right]}\right| .
$$

For the particular case $A=0.5$, corresponding to a completely unpolarized initial source, the latter equation reduces to

$\mathscr{P}_{\mathrm{I}}^{\infty}(A=0.5)=\left|\frac{\tilde{I}\left(\frac{1}{L}\right)}{\tilde{I}(0)}\right|$.

It is seen that the degree of polarization depends only on the ratio between the Fourier transform of the intensity profiles evaluated at $1 / L$ and 0 , respectively. It is worthwhile to note that $\mathscr{P}_{\mathrm{I}}^{\infty}$ does not depend on the degree of coherence of the source and assumes one and the same value everywhere across the transversal section of the beam. This means that in the far zone we obtain a partially and uniformly polarized beam. It can be noted that by changing the period of the grating it is possible to obtain any value of the degree of polarization.

Further significant cases are $A=1$ and $A=0$, corresponding to fully linearly polarized initial sources, along the $x$ - and $y$-axis, respectively. For $A=1$ we have

$$
\mathscr{P}_{\mathrm{I}}^{\infty}(A=1)=\left|\frac{\tilde{I}(0)+\tilde{I}\left(\frac{2}{L}\right)+2 \tilde{I}\left(\frac{1}{L}\right)}{2\left[\tilde{I}(0)+\tilde{I}\left(\frac{1}{L}\right)\right]}\right|,
$$

while, for $A=0$,

$$
\mathscr{P}_{\mathrm{I}}^{\infty}(A=0)=\left|\frac{\tilde{I}(0)+\tilde{I}\left(\frac{2}{L}\right)-2 \tilde{I}\left(\frac{1}{L}\right)}{2\left[\tilde{I}(0)-\tilde{I}\left(\frac{1}{L}\right)\right]}\right| .
$$

Note that $\mathscr{P}_{\mathrm{I}}^{\infty}$ does not depend on the coordinate $p$, so that the depolarization effect is uniform across the transversal section of the beam in the far field. 
Information about the polarization state of the beam in the far zone is contained in the Stokes parameters, which are given by (see Eqs. (5)-(8))

$s_{0, \mathrm{I}}^{\infty}(p)=\frac{\tilde{\mu}(p)}{2}\left[\tilde{I}(0)+(2 A-1) \tilde{I}\left(\frac{1}{L}\right)\right]$,

$s_{1, \mathrm{I}}^{\infty}(p)=\frac{\tilde{\mu}(p)}{4}(2 A-1)\left[\tilde{I}(0)+\tilde{I}\left(\frac{2}{L}\right)\right]+2 \tilde{I}\left(\frac{1}{L}\right)$,

$s_{2, \mathrm{I}}^{\infty}(p)=0$,

$s_{3, \mathrm{I}}^{\infty}(p)=0$.

Eqs. (38)-(41) say that the state of polarization changes with the coordinate $p$, and its value depends on both the coherence function and the intensity profile of the source. The totally polarized component [23] is always linear with constant azimuth $\alpha=0$. This result is related to the structure of the PG, which has a horizontal transmission axis in the center. Analogous results could be obtained for the cases of linearly polarized sources with different azimuths. Those cases reduce to the previous ones by suitably shifting the PG along the $x$-axis.

\subsection{Case II: Superposition of circularly polarized} sources

In this case the partially coherent partially polarized source is represented by the following BCP matrix:

$$
\begin{aligned}
\hat{J}_{\mathrm{II}}\left(x_{1}, x_{2}\right)= & \frac{1}{2} J_{\mathrm{L}}\left(x_{1}, x_{2}\right)\left(\begin{array}{cc}
1 & -\mathrm{i} \\
\mathrm{i} & 1
\end{array}\right) \\
& +\frac{1}{2} J_{\mathrm{R}}\left(x_{1}, x_{2}\right)\left(\begin{array}{cc}
1 & \mathrm{i} \\
-\mathrm{i} & 1
\end{array}\right),
\end{aligned}
$$

where $J_{\mathrm{L}}$ and $J_{\mathrm{R}}$ are chosen as

$$
J_{\mathrm{L}}\left(x_{1}, x_{2}\right)=A J_{\mathrm{s}}\left(x_{1}, x_{2}\right) ; \quad J_{\mathrm{R}}\left(x_{1}, x_{2}\right)=B J_{\mathrm{s}}\left(x_{1}, x_{2}\right),
$$

so that

$$
\hat{J}_{\mathrm{II}}\left(x_{1}, x_{2}\right)=\frac{1}{2} J_{\mathrm{s}}\left(x_{1}, x_{2}\right)\left(\begin{array}{cc}
A+B & -\mathrm{i}(A-B) \\
\mathrm{i}(A-B) & A+B
\end{array}\right) .
$$

The condition (14), i.e., $A+B=1$, is still required for the coefficients $A$ and $B$. Again, the intensity of the resulting source is unchanged on varying $A$ but the choice of $A$ sets the degree polarization of the source. The latter is completely unpolarized if $A=B=0.5$ and is fully circularly polarized when $A=1$ (left-handed) or $A=0$ (right-handed). The expression of the degree of polarization turns out to be the same as Eq. (15).

When the source is covered by the polarization grating described by the Jones matrix in Eq. (16), the BCP matrix of the emerging field takes the form

$$
\begin{aligned}
\hat{J}\left(x_{1}, x_{2}\right)=J_{\mathrm{s}}\left(x_{1}, x_{2}\right)\left\{\frac{A}{2} \exp \left[-\mathrm{i} \frac{\pi}{L}\left(x_{1}-x_{2}\right)\right]\right. & \\
& \left.+\frac{B}{2} \exp \left[\mathrm{i} \frac{\pi}{L}\left(x_{1}-x_{2}\right)\right]\right\}\left(\begin{array}{ll}
C_{1} C_{2} & C_{1} S_{2} \\
C_{2} S_{1} & S_{1} S_{2}
\end{array}\right) .
\end{aligned}
$$

It is easily seen that even in this case the degree of polarization is one at any point at the output of the polarization grating. The difference with the previous case lies in the fact that now the intensity profile across the output plane is unaffected by the presence of the polarizer. From the general relation (3) in fact we have

$I(x)=I_{\mathrm{s}}(x) \frac{A+B}{2}=\frac{1}{2} I_{\mathrm{s}}(x)$.

The evaluation of the BCP matrix in the far field yields

$$
\begin{aligned}
& J_{x x}^{\infty}(P, Q)= \\
& \quad \frac{K}{8}\left\{\tilde{I}(Q) \tilde{\mu}(P)+A\left[\tilde{\mu}\left(P-\frac{1}{L}\right)-\tilde{\mu}\left(P+\frac{1}{L}\right)\right]\right. \\
& \quad+A\left[\tilde{I}\left(Q-\frac{1}{L}\right)+\tilde{I}\left(Q+\frac{1}{L}\right)\right] \\
& \quad \times\left[\tilde{\mu}\left(P-\frac{1}{2 L}\right)-\tilde{\mu}\left(P+\frac{1}{2 L}\right)\right] \\
& \quad+\left[\tilde{I}\left(Q-\frac{1}{L}\right)+\tilde{I}\left(Q+\frac{1}{L}\right)\right] \\
& \left.\quad \times \tilde{\mu}\left(P+\frac{1}{2 L}\right)+\tilde{I}(Q) \tilde{\mu}\left(P+\frac{1}{L}\right)\right\},
\end{aligned}
$$




$$
\begin{aligned}
& J_{x y}^{\infty}(P, Q)= \\
& \quad \frac{K}{8 \mathrm{i}}\left\{A\left[\tilde{I}\left(Q+\frac{1}{L}\right)-\tilde{I}\left(Q-\frac{1}{L}\right)\right]\right. \\
& \quad \times\left[\tilde{\mu}\left(P-\frac{1}{2 L}\right)-\tilde{\mu}\left(P+\frac{1}{2 L}\right)\right] \\
& \quad-\tilde{I}(Q)\left[(2 A-1) \tilde{\mu}(P)+(1-A) \tilde{\mu}\left(P+\frac{1}{L}\right)\right. \\
& \left.\quad-A \tilde{\mu}\left(P-\frac{1}{L}\right)\right]+\left[\tilde{I}\left(Q+\frac{1}{L}\right)\right. \\
& \left.\left.\quad-\tilde{I}\left(Q-\frac{1}{L}\right)\right] \tilde{\mu}\left(P+\frac{1}{2 L}\right)\right\},
\end{aligned}
$$

$$
\begin{aligned}
& J_{y x}^{\infty}(P, Q)= \\
& \quad \frac{K}{8 \mathrm{i}}\left\{A\left[\tilde{I}\left(Q+\frac{1}{L}\right)-\tilde{I}\left(Q-\frac{1}{L}\right)\right]\right. \\
& \quad \times\left[\tilde{\mu}\left(P-\frac{1}{2 L}\right)-\tilde{\mu}\left(P+\frac{1}{2 L}\right)\right] \\
& \quad+\tilde{I}(Q)\left[(2 A-1) \tilde{\mu}(P)+(1-A) \tilde{\mu}\left(P+\frac{1}{L}\right)\right. \\
& \left.\quad-A \tilde{\mu}\left(P-\frac{1}{L}\right)\right]+\left[\tilde{I}\left(Q+\frac{1}{L}\right)\right. \\
& \left.\left.\quad-\tilde{I}\left(Q-\frac{1}{L}\right)\right] \tilde{\mu}\left(P+\frac{1}{2 L}\right)\right\},
\end{aligned}
$$

$$
\begin{aligned}
& J_{y y}^{\infty}(P, Q)= \\
& \quad \frac{K}{8}\left\{\tilde{I}(Q) \tilde{\mu}(P)+A \tilde{I}(Q)\left[\tilde{\mu}\left(P-\frac{1}{L}\right)-\tilde{\mu}\left(P+\frac{1}{L}\right)\right]\right. \\
& \quad-A\left[\tilde{I}\left(Q-\frac{1}{L}\right)+\tilde{I}\left(Q+\frac{1}{L}\right)\right] \\
& \quad \times\left[\tilde{\mu}\left(P-\frac{1}{2 L}\right)-\tilde{\mu}\left(P+\frac{1}{2 L}\right)\right] \\
& \quad-\left[\tilde{I}\left(Q-\frac{1}{L}\right)+\tilde{I}\left(Q+\frac{1}{L}\right)\right] \\
& \left.\quad \times \tilde{\mu}\left(P+\frac{1}{2 L}\right)+\tilde{I}(Q) \tilde{\mu}\left(P+\frac{1}{L}\right)\right\},
\end{aligned}
$$

and the degree of polarization in the far field can be calculated by Eq. (4).

The simplifying hypotheses made in the previous subsection about the functions $\tilde{\mu}$ and $\tilde{I}_{\mathrm{s}}$, i.e.,

$$
\begin{aligned}
\tilde{\mu}\left(Q-\frac{1}{L}\right) & \simeq \tilde{\mu}\left(Q+\frac{1}{L}\right) \simeq \tilde{\mu}(Q) \text { and } I_{\mathrm{s}}(x) \\
& =I_{\mathrm{s}}(-x),
\end{aligned}
$$

finally lead to

$$
\begin{aligned}
& J_{x x}^{\infty}(p, 0) \simeq \frac{\tilde{\mu}(p)}{4}\left[\tilde{I}(0)+\tilde{I}\left(\frac{1}{L}\right)\right], \\
& J_{y y}^{\infty}(p, 0) \simeq \frac{\tilde{\mu}(p)}{4}\left[\tilde{I}(0)-\tilde{I}\left(\frac{1}{L}\right)\right], \\
& J_{x y}^{\infty}(p, 0) \simeq 0, \\
& J_{y x}^{\infty}(p, 0) \simeq 0,
\end{aligned}
$$

whence

$$
\mathscr{P}_{\text {II }}^{\infty}(A)=\left|\frac{\tilde{I}\left(\frac{1}{L}\right)}{\tilde{I}(0)}\right| .
$$

This expression coincides with the degree of polarization for a unpolarized source, that is $P_{\mathrm{I}}^{\infty}(A=$ $0.5)$ (see Eq. (35)). Note that the expression in Eq. (55) does not depend on $A$ : this means that, under the hypotheses (i) and (ii) done before, the degree of polarization has the same form for all the sources belonging to the considered class, irrespective of their degree of polarization. Furthermore, from Eq. (55) it is clear that, in order to obtain a completely unpolarized beam at the far field, it is necessary that $\tilde{I}$ assumes sufficiently small values when its argument is $1 / L$.

Similarly as we have done for the case I, the state of polarization in the far zone is expressed through the Stokes parameters of the field. From Eqs. (5)-(8) we have

$$
\begin{aligned}
s_{0, \mathrm{II}}^{\infty}(p) & =\frac{1}{2} \tilde{\mu}(p) \tilde{I}(0), \\
s_{1, \mathrm{II}}^{\infty}(p) & =\frac{1}{2} \tilde{\mu}(p) \tilde{I}\left(\frac{1}{L}\right), \\
s_{2, \mathrm{II}}^{\infty}(p) & =0, \\
s_{3, \mathrm{II}}^{\infty}(p) & =0 .
\end{aligned}
$$

The state of polarization, as in the previous case, depends on the degree of coherence and on the intensity of the initial source. The totally polarized 
component is still linearly polarized along the $x$ axis.

\section{Gaussian Schell-model sources}

In the present section we specialize the general analysis presented in Section 3 to the case of a particular form of the correlation function $J_{\mathrm{s}}\left(x_{1}, x_{2}\right)$, namely, the so-called GSM source, for which the intensity profile and the degree of coherence are both Gaussian, i.e.,

$$
\left\{\begin{array}{l}
I_{\mathrm{s}}(x)=I_{0} \exp \left(-\frac{x^{2}}{2 \sigma_{I}^{2}}\right), \\
\mu(x)=\exp \left(-\frac{x^{2}}{2 \sigma_{\mu}^{2}}\right)
\end{array}\right.
$$

where $I_{0}$ is a constant intensity factor, while $\sigma_{I}^{2}$ and $\sigma_{\mu}^{2}$ are the variances of the transverse intensity profile and of the degree of coherence, respectively. It should be noted that GSM sources are of the Q$\mathrm{H}$ type only if $\sigma_{\mu} \ll \sigma_{I}$. Nonetheless, for any choice of $\sigma_{\mu}$ and $\sigma_{I}$, their mutual intensity can be always written in the form (25), but the functions $I_{\mathrm{s}}$ and $\mu$ in Eq. (25) no longer represent the intensity profile and the degree of coherence respectively.

The Fourier transforms of the latter functions are

$\left\{\begin{array}{l}\tilde{I}(p)=I_{0} \sqrt{2 \pi \sigma_{I}^{2}} \exp \left(-2 \pi^{2} \sigma_{I}^{2} p^{2}\right), \\ \tilde{\mu}(p)=\sqrt{2 \pi \sigma_{\mu}^{2}} \exp \left(-2 \pi^{2} \sigma_{\mu}^{2} p^{2}\right),\end{array}\right.$

respectively. The hypothesis (i) and Eq. (61) require that

$\frac{2}{L} \ll \frac{1}{2 \pi \sigma_{\mu}}$,

which means that the width of the degree of coherence is smaller than the period of the grating, while hypothesis (ii) is ensured by the analytical form of $I_{\mathrm{s}}(x)$.

In the following we limit ourselves to study the degree of polarization of the beam in the far field for the case of sources obtained as incoherent superpositions of linearly polarized sources. The analogous quantity for the other case (superposition of circularly polarized sources) is simply obtained on letting $A=0.5$ in the previous one, because $P_{\mathrm{II}}^{\infty}(A)=P_{\mathrm{I}}^{\infty}(A=0.5)$. The expression of the degree of polarization, $P_{\mathrm{I}}^{\infty}(A)$, which can be calculated from Eqs. (34) and (61) turns out to be $\mathscr{P}_{\mathrm{I}}^{\infty}(A)=$

$$
\frac{1}{2}\left|\frac{(2 A-1)\left[1+\exp \left(-\frac{8 \pi^{2} \sigma_{I}^{2}}{L^{2}}\right)\right]+2 \exp \left(-\frac{2 \pi^{2} \sigma_{I}^{2}}{L^{2}}\right)}{1+(2 A-1) \exp \left(-\frac{8 \pi^{2} \sigma_{I}^{2}}{L^{2}}\right)}\right|,
$$

which depends only on $A$ and on the ratio $L / \sigma_{I}$. For $L \ll \sigma_{I}$ this expression tends to the value $\mathscr{P}_{\mathrm{s}} / 2$.

On the other hand, for the case $A=0.5$, Eq. (63) reduces to

$$
\mathscr{P}_{\mathrm{I}}^{\infty}(A=0.5)=\mathscr{P}_{\mathrm{II}}^{\infty}(A)=\exp \left(-\frac{2 \pi^{2} \sigma_{I}^{2}}{L^{2}}\right),
$$

so that the beam turns out to be completely unpolarized when the condition

$\sigma_{I} \gg L$

is met. A simple way to interpret this depolarization effect is the following: Let us consider a point far away from the source. In this point the field is the sum of many contributions, each of them corresponding to an infinitesimal element of the source. The different contributions are mutually uncorrelated (see Eq. (62)) and have approximately the same weight. Moreover, due to condition (65) the polarization grating provides all possible linear polarization states with the same intensity. As a consequence, at the far field the beam is a superposition of equal and independent contributions, all of them linearly polarized with all possible azimuths, giving rise to a completely unpolarized beam. The polarization degree turns out to be one half of that pertinent to the initial source. This, in general, does not happen for different values of $A$, because in that case the contributions of different polarization states have not the same intensity. In Fig. 2 the degree of polarization is plotted versus $L / \sigma_{I}$. Depending on the value of $A, \mathscr{P}_{\mathrm{I}}^{\infty}$ exhibits two different behaviors. For $A \geqslant 0.5$ the degree of polarization increases as $L / \sigma_{I}$ increases, reaching its minimum value $\left(\mathscr{P}_{\mathrm{s}} / 2\right)$ when $L=0$. In practice, such value is reached for $L / \sigma_{I} \lesssim 2$, and is zero for any incoherent superposition of circularly polarized sources. In the opposite limit, a completely 


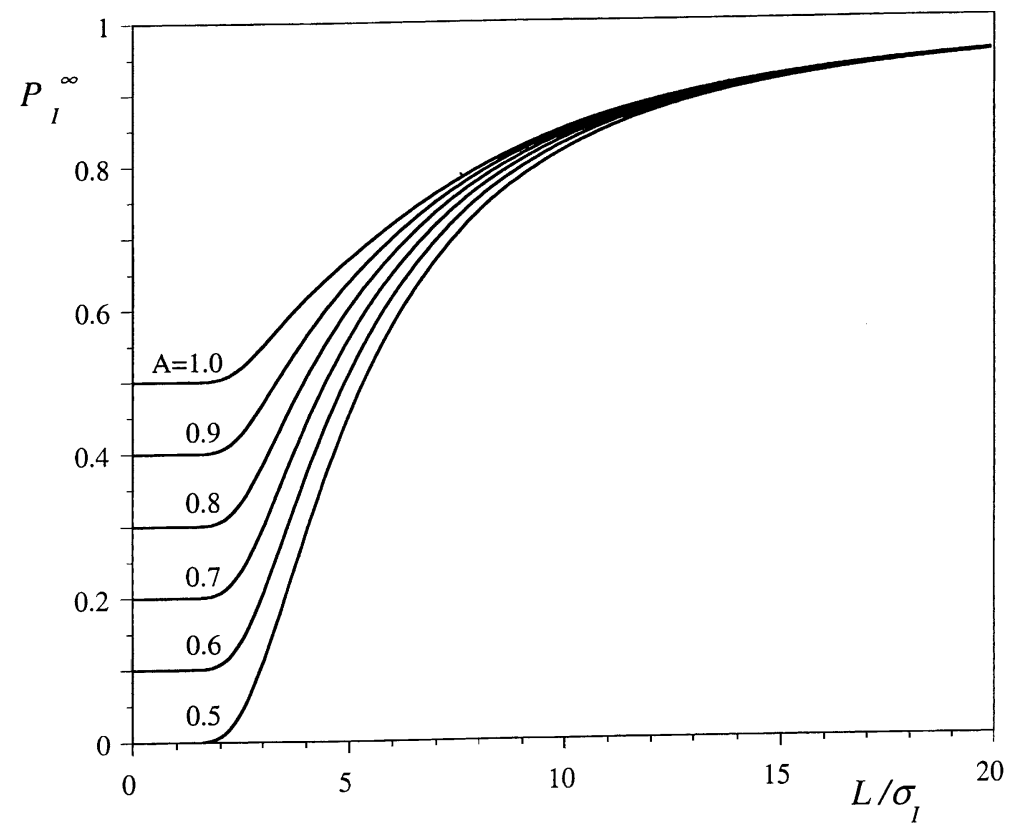

Fig. 2. Degree of polarization $\mathscr{P}_{\mathrm{I}}^{\infty}$ versus $L / \sigma_{I}$ for different values of $A \geqslant 0.5$.

polarized beam is always obtained in the far zone. In such a limit, indeed, the PG behaves like a simple linear polarizer.

In Fig. 3 the cases with $A \leqslant 0.5$ are shown. For the limiting cases $L \rightarrow 0$ and $L \rightarrow \infty$ the same considerations as for the previous figures hold. An interesting feature of the present case is the fact that a particular value of $L / \sigma_{I}$, depending on $A$, exists such that $\mathscr{P}_{\mathrm{I}}^{\infty}=0$. In order to explain it in a simple way, let us consider the case of a fully $y$ polarized $(A=0)$ Gaussian source having width $\sigma_{I}$. The intensity beyond the PG has a null at the center of the beam $(x=0)$. It may happen that the beam emerging from the PG will present two lobes, approximately polarized along orthogonal directions, which contribute to the far field in such a way that the resulting degree of polarization in the far zone turns out to be zero.

\section{Conclusions}

In the present paper the polarization features of beams emitted by a class partially polarized Q-H sources and passing through PGs have been studied. It has been shown how degree of polarization and Stokes parameters at the far zone depend on the source characteristics, such as its state of polarization, intensity, and degree of coherence, and on the period of the grating. It turns out that in the far zone the beam is, in general, partially and non-uniformly polarized across its transversal section. However, if the coherent length of the Q-H source is made smaller than the grating period and the intensity profile is represented by an even function, simple closed-form expressions of the above parameters can be given. Furthermore in such a case the degree of polarization turns out to be uniform across the section of the beam and on varying the period of the grating can take any value from zero to one. It suggests a way for synthesizing completely and uniformly non-polarized beams. Different states of polarization of the initial sources have been considered. In particular, it has been shown that for any incoherent superposition of orthogonally circularly polarized sources the same degree of polarization in the far zone is obtained. On the contrary, when orthogonally linearly polarized uncorrelated sources are considered, the results not only depend on the 


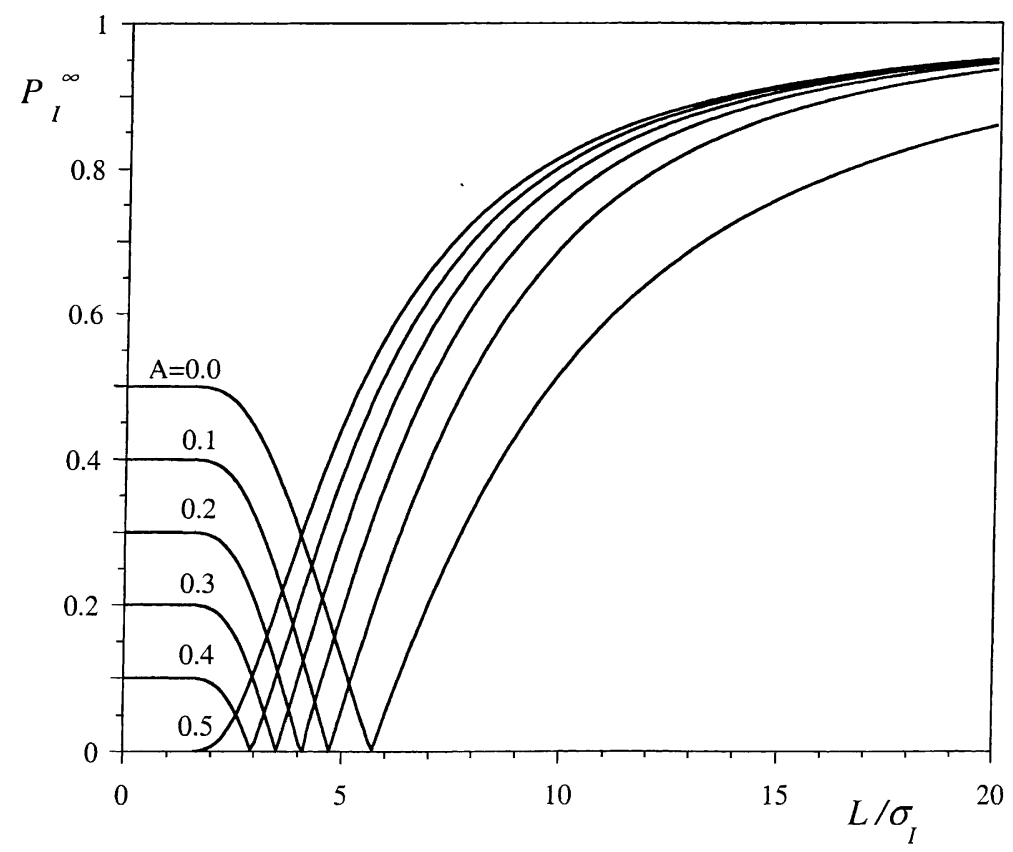

Fig. 3. Degree of polarization $\mathscr{P}_{\mathrm{I}}^{\infty}$ versus $L / \sigma_{I}$ for different values of $A \leqslant 0.5$.

period of the grating but also on the intensities of the two orthogonal components of the electric field. Finally, the example of a GSM beam clarified the meaning of the assumed hypotheses and allowed numerical results to be presented.

\section{Acknowledgements}

We wish to thank Franco Gori for the helpful discussions and his valuable suggestions. We also acknowledge the project Acción Integrada Hispano-Italiana HI-1998-077. One of the authors (G.P.) thanks the support from a grant "Ayudas Complutense postdoctorales en el extranjero" and the project PB97-295.

\section{References}

[1] S.C. Tidwell, D.H. Ford, W.D. Kimura, Generating radially polarized beams interferometrically, Appl. Opt. 29 (1990) 2234-2239.
[2] T. Erdogan, O. King, G.W. Wicks, D.G. Hall, E. Anderson, M.J. Rooks, Circularly symmetric operation of a concentric-circle-grating surface emitting AlGaAs/GaAs quantum well semiconductor laser, Appl. Phys. Lett. 60 (1992) 1921-1923.

[3] P.L. Greene, D.G. Hall, Properties and diffraction of vector Bessel-Gauss beams, J. Opt. Soc. Am. A 15 (1998) 3020-3027.

[4] A.A. Tovar, Production and propagation of cylindrically polarized Laguerre Gaussian laser beams, J. Opt. Soc. Am. A 15 (1998) 2705-2711.

[5] J.M. Movilla, G. Piquero, R. Martínez-Herrero, P.M. Mejías, Parametric characterization of non-uniformly polarized beams, Opt. Commun. 149 (1998) 230234.

[6] A.K. Jaiswal, G.P. Agrawal, C.L. Mehta, Coherence functions in the far-field diffraction plane, Il Nuovo Cimento 15B (1973) 295-307.

[7] D.F.V. James, Polarization of light radiated by black-body sources, Opt. Commun. 109 (1994) 209-214.

[8] F. Gori, M. Santarsiero, R. Borghi, G. Piquero, Use of van Cittert-Zernike theorem for partially polarized sources, Opt. Lett. 25 (2000) 1291-1293.

[9] D.F.V. James, Change of polarization of light beams on propagation in free space, J. Opt. Soc. Am. A 5 (1994) 1641-1643.

[10] G.P. Agrawal, E. Wolf, Propagation-induced polarization changes in partially coherent optical beams, J. Opt. Soc. Am. A 17 (2000) 2019-2023. 
[11] F. Gori, M. Santarsiero, G. Piquero, R. Borghi, A. Mondello, R. Simon, Partially polarized Gaussian Schellmodel beams, J. Opt. A: Pure Appl. Opt. 3 (2001) 1-9.

[12] F. Gori, Measuring Stokes parameters by means of a polarization grating, Opt. Lett. 24 (1999) 584-586.

[13] C.G. Someda, Far field of polarization gratings, Opt. Lett. 25 (2000) 1657-1659.

[14] P. Rochon, V. Drnoyan, A. Natansohn, Polarization holographic gratings in azopolymers for detecting and producing circularly polarized light, in: G.A. Lampropoulos, R.A. Lessard (Eds.), Proceedings of the 1998 International Conference on applications of photonic technology. III. Closing the gap between theory, developments, and applications, SPIE 3491, 2000, pp. 306-309.

[15] J. Tervo, J. Turunen, Paraxial-domain diffractive elements with $100 \%$ efficiency based on polarization gratings, Opt. Lett. 25 (2000) 785-786.

[16] F. Gori, Matrix treatment for partially polarized, partially coherent beams, Opt. Lett. 23 (1998) 241-243.
[17] F. Gori, M. Santarsiero, S. Vicalvi, R. Borghi, G. Guattari, Beam coherence-polarization matrix, J. Eur. Opt. Soc. A: Pure Appl. Opt. 7 (1998) 941-951.

[18] L. Mandel, E. Wolf, Optical Coherence and Quantum Optics, Cambridge University Press, Cambridge, 1995.

[19] E. Collett, E. Wolf, Is complete coherence necessary for the generation of highly directional light beams? Opt. Lett. 2 (1978) 27-29.

[20] F. Gori, C. Palma, Partially coherent sources which give rise to highly directional light beams, Opt. Commun. 27 (1978) 185-188.

[21] W.K. Burns, R.P. Moeller, C.H. Bulmer, A.S. Greenblatt, Depolarized source for fiber-optic applications, Opt. Lett. 16 (1991) 381-383.

[22] W. Jin, G. Stewart, K. Crawford, B. Culshaw, Fiber-optic pseudodepolarizer based on birefringence modulation, Opt. Lett. 20 (1995) 1737-1739.

[23] M. Born, E. Wolf, Principles of Optics, seventh edition, Cambridge University Press, Cambridge, 1999. 